LUNG CANCER

\section{Every cell counts}

Peripheral blood circulating tumour cells (CTCs) have tumorigenic potential in vivo and have prognostic significance in patients with several types of cancer. Patients with earlystage non-small-cell lung cancer (NSCLC) have a $50 \%$ risk of relapse within 5 years after surgery, thus strategies to identify patients with high risk of recurrence are needed. However, peripheral blood CTCs are rare in patients with early-stage NSCLCs. Now, Chemi at al. show that pulmonary venous circulating tumour cells (PV-CTCs), detected based on EpCAM and cytokeratin expression using CellSearch, are present in patients with NSCLC at tumour resection, and the PV-CTC count was associated with lung cancer-specific relapse.

Out of a cohort of 100 patients with NSCLC from the tracking cancer evolution through therapy $(\mathrm{Rx})$ (TRACERx) study, prospectively recruited at clinical sites where PV-CTC collection at the stage of tumour resection was possible, 48 patients had at least $1 \mathrm{PV}-\mathrm{CTC}$ per $7.5 \mathrm{ml}$ of blood. Although PV-CTC count was not associated with pathological stage, smoking status or other clinicopathological factors, high counts of PV-CTCs (at least 7 PV-CTCs per $7.5 \mathrm{ml}$ of blood) were associated with lung cancer relapse within 2 years of surgery. Moreover, each doubling of the PV-CTC count was a significant prognostic factor for disease-free survival (DFS) and was associated with lung cancer-specific relapse in univariate and multivariate analysis.

The researchers then focused on one patient to see how the genomic profiles of primary tumour cells, early-disseminated PV-CTCs and cells from metastatic disease compared. Among the 14 patients in whom PV-CTC and control cell isolation had been successful, only 1 patient also had an evaluable metastatic tissue biopsy. This patient had been diagnosed with relapsed disease 10 months after surgery and metastases in the brain shortly afterwards, and died a few months later after having received palliative chemotherapy and radiotherapy. In this patient, $28 \mathrm{PV}-\mathrm{CTC}$ had been detected, 6 of which were then successfully isolated and amplified by the researchers. Low-pass wholegenome sequencing detected copy number changes that matched the primary tumour in 3 out of 6 PV-CTCs. The remaining cells had control-like genomic profiles, leading the researchers to label these normal-like circulating epithelial cells (CECs).

Following whole-exome sequencing (WES), the researchers identified 198 mutations in PV-CTCs (none was found in CECs). Of those 198 mutations, 91\% were also present in metastases, and $79 \%$ were also present in the primary tumour. Conversely, $49 \%$ of primary tumour mutations were found in PV-CTCs, whereas $97 \%$ of primary tumour mutations

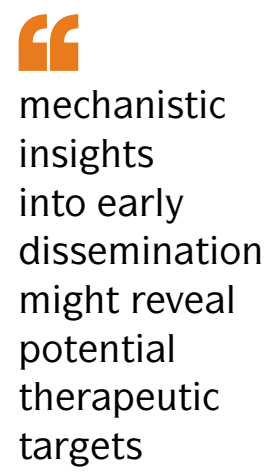

targets

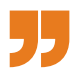

not found in PV-CTCs were also no present in metastases. Phylogenetic analysis confirmed that PV-CTCs and metastatic cells share the same specific branch distinct from all other primary tumour subclones, indicating a common progenitor, and which represents a tumour subclone likely responsible for relapse.

Targeted deep sequencing revealed that 13 mutations initially found only in PV-CTCs were also present in the metastatic and/or primary tumour and/or relapse cell-free (cf) DNA, but were not detected in pulmonary or peripheral blood cfDNA samples collected at resection. The phylogenetic connection between PV-CTCs and metastases opens up opportunities to explore the molecular mechanisms of metastatic spread in NSCLC. PV-CTCs can be detected as early as 10 months before overt metastases, so mechanistic insights into early dissemination might reveal potential therapeutic targets and strategies to prevent metastatic spread. Indeed, the authors identified a putative inactivating driver mutation in the tumour suppressor gene LZTS1 that overlapped between PV-CTCs and metastases but was not detected in the primary tumour.

These results highlight the potential of using PV-CTCs from patients with NSCLC to predict recurrence. Of note, the performance using a cut-off point of at least 7 PV-CTCs per $7.5 \mathrm{ml}$ of blood in predicting relapse was higher than when using a cut-off point of at least 18 as previously published by the authors, although this approach was similarly low in sensitivity. The modest predictive strength might also be confounded by the presence of CECs in the blood samples. Therefore, before the application of PV-CTCs as predictors of recurrence can benefit patients with early-stage NSCLC more detailed analyses of the circulating cell populations are warranted.

Ulrike Harjes ORIGINAL ARTICLE Chemi, F. et al. Pulmonary venous circulating tumor cell dissemination before tumor resection and disease relapse. Nat. Med. 25 , 1534-1539 (2019) RELATED ARTICLE Keller, L. \& Pantel, K. Unravelling tumour heterogeneity by single-cell profiling of circulating tumour cells. Nat. Rev. Cancer 19, 553-567 (2019) 